\title{
Calidad de semen bovino diluido y congelado en un diluyente con caseinato de sodio
}

\author{
Quality of bovine semen diluted and frozen in an extender with sodium caseinate
}

Jackeline Pérez ${ }^{1}$, Giovanni Restrepo B. ${ }^{2}$, Alexandra Usuga S. ${ }^{1,3}$

\section{RESUMEN}

\begin{abstract}
El objetivo del estudio fue evaluar el caseinato de sodio (CS) en la dilución y la congelación de semen bovino. Quince eyaculados provenientes de cinco toros fueron diluidos y congelados individualmente con un diluyente compuesto por tris-glicerolácido cítrico con $8 \% \mathrm{v} / \mathrm{v}$ de lipoproteínas de baja densidad (LDL) y suplementado con CS en cuatro tratamientos: CS 3\% p/v (CS3), CS 4\% p/v (CS4), CS 5\% p/v (CS5) y sin CS (Control). Se evaluaron la movilidad y cinética (Sistema $S_{C A}{ }^{\circledR}$ ) y la integridad funcional de membrana (prueba HOS) del semen fresco, diluido y descongelado. Además, se evaluó la morfología y vitalidad (eosina-nigrosina) del semen fresco y diluido, y la integridad estructural de membrana (SYBR14/IP), la integridad acrosómica (FITC-PNA) y la capacidad fertilizante in vitro (FIV) del semen descongelado. El análisis estadístico de los parámetros de calidad espermática se realizó mediante modelos mixtos y la comparación de las medias por la prueba de Tukey. El análisis estadístico de la FIV se realizó con la prueba de Kruskal-Wallis. CS3 produjo un incremento de la movilidad progresiva y la frecuencia de batido del semen descongelado $(\mathrm{p}<0.05)$. CS4 tuvo un efecto deletéreo sobre la movilidad progresiva del semen diluido, mientras CS4 y CS5 afectaron la movi-
\end{abstract}

${ }^{1}$ Facultad de Medicina Veterinaria y Zootecnia, Universidad CES, Medellín, Colombia

${ }^{2}$ Facultad de Ciencias Agrarias, Universidad Nacional de Colombia sede Medellín, Medellín, Colombia

${ }^{3}$ E-mail: ausuga@ces.edu.co

Recibido: 14 de abril de 2020

Aceptado para publicación: 10 de noviembre de 2021

Publicado: 22 de diciembre de 2021

CLos autores. Este artículo es publicado por la Rev Inv Vet Perú de la Facultad de Medicina Veterinaria, Universidad Nacional Mayor de San Marcos. Este es un artículo de acceso abierto, distribuido bajo los términos de la licencia Creative Commons Atribución 4.0 Internacional (CC BY 4.0) [https:// creativecommons.org/licenses/by/4.0/deed.es] que permite el uso, distribución y reproducción en cualquier medio, siempre que la obra original sea debidamente citada de su fuente original 
lidad, cinética e integridad de membrana del semen descongelado ( $p<0.05$ ). No se encontraron diferencias para la FIV ( $\mathrm{p}>0.05)$. La suplementación del diluyente de congelación con caseinato de sodio puede mejorar la calidad posdescongelación del semen bovino.

Palabras clave: bovinos, caseinato de sodio, criopreservación, diluyente, semen

\section{Abstract}

The aim of this study was to evaluate sodium caseinate (CS) in the dilution and freezing of bovine semen. Fifteen ejaculates from five bulls were diluted and then individually frozen using an extender composed of tris-glycerol-citric acid with $8 \% \mathrm{v} / \mathrm{v}$ of low-density lipoproteins (LDL) and supplemented with CS in four treatments: CS 3\% w/ $\mathrm{v}(\mathrm{CS} 3), \mathrm{CS} 4 \% \mathrm{w} / \mathrm{v}(\mathrm{CS} 4), \mathrm{CS} 5 \% \mathrm{w} / \mathrm{v}(\mathrm{CS} 5)$ and without CS (Control). The motility and kinetics (SCA® System) and the functional integrity of the membrane (HOS test) of the fresh, extended and thawed semen were evaluated. In addition, the morphology and vitality (eosin-nigrosin) of the fresh and extended semen, and the structural integrity of the membrane (SYBR14/IP), the acrosome integrity (FITC-PNA) and the in vitro fertilizing capacity (IVF) of thawed semen were evaluated. The statistical analysis of the sperm quality parameters was performed using mixed models and the comparison of the means by the Tukey test. Statistical analysis of IVF was performed with the Kruskal-Wallis test. CS3 produced an increase in progressive motility and beat cross frequency of thawed semen $(\mathrm{p}<0.05)$. CS4 had a deleterious effect on progressive motility of extended semen, while CS4 and CS5 affected the motility, kinetics and membrane integrity of thawed semen $(p<0.05)$. No differences were found for IVF ( $p>0.05)$. Supplementation of freezing extender with sodium caseinate can improve post-thaw quality of bovine semen.

Key words: bovine, sodium caseinate, cryopreservation, extender, semen

\section{INTRODUCCIÓN}

El uso de crioprotectores reduce los efectos perjudiciales de la congelación y descongelación sobre los espermatozoides, ya que los protege del choque térmico, el estrés osmótico y las alteraciones en la fluidez y la permeabilidad de la membrana (Murphy et al., 2017). A pesar de los buenos resultados obtenidos con crioprotectores como la yema de huevo y la leche (Bergeron y Manjunath 2006), existe un énfasis creciente en la necesidad de eliminar productos de origen animal de los diluyentes de congelación, con el fin de facilitar la comercialización internacional de semen congelado (Layek et al., 2016; Anzar et al., 2019). Además, éstos presen- tan una alta variabilidad en sus componentes (Plante et al., 2015), interfieren y dificultan la evaluación microscópica y los ensayos metabólicos y bioquímicos en el semen diluido (Singh et al., 2012, 2013), y pueden alterar la integridad estructural y funcional de los espermatozoides descongelados (Pugliesi et al., 2012). Así mismo, se ha reportado que los productos de origen animal pueden presentar endotoxinas que reducen la capacitad fertilizante de los espermatozoides (Aires et al., 2003), y que microorganismos como Escherichia coli, Staphylococcus, Streptococcus, Pseudomonus, Haemophilus, Salmonella, Campylobacter, Listeria y Mycoplasma, así como el virus de la influenza aviar pueden ser transmitidos por la yema de huevo (Thibier y Guerin, 2000). 
Entre las alternativas para reemplazar la yema de huevo y la leche en los diluyentes de congelación de semen bovino se destaca el uso de lecitinas de soya (Aires et al., 2003); sin embargo, los diluyentes basados en este componente varían bastante entre lotes y producen tasas de fertilidad inconsistentes (Van Wagtendonk-de Leeuw et al., 2000; Layek et al., 2016). Por lo tanto, se ha recurrido al uso de componentes lipídicos purificados como el colesterol o los liposomas, los cuales han mostrado una capacidad equivalente de protección espermática a la obtenida con los diluyentes convencionales de congelación; sin embargo, su uso comercial se ha visto limitado por su alto costo (Röpke et al., 2011; Kumar et al., 2015; Anzar et al., 2019). Las lipoproteínas de baja densidad (LDL) purificadas han sido otra alternativa para reemplazar la yema de huevo en los diluyentes de congelación, alcanzando niveles de protección espermática equivalentes o superiores a la yema de huevo entera (Moussa et al., 2002; Varela et al., 2020a).

El caseinato de sodio (CS) es un aislado de caseína que se obtiene mediante la precipitación de las caseínas de la leche y su neutralización con hidróxido sódico, que por su producción estandarizada podría ser una alternativa de origen animal para la congelación de semen bovino. Es probable que el componente crioprotector de la leche sean las micelas de caseína, que son sus principales proteínas (Bergeron et al., 2007). Mediante estudios previos se ha demostrado que las micelas de caseína pueden proteger el semen bovino, equino y de pequeños rumiantes durante el almacenamiento a $4{ }^{\circ} \mathrm{C}$ (Batellier et al., 1997; Bergeron et al., 2007). El objetivo de este estudio fue evaluar el uso de caseinato de sodio (CS) en la dilución y la congelación del semen bovino, y su efecto sobre la calidad y la capacidad fertilizante in vitro de los espermatozoides.

\section{Materiales y MéTodos}

\section{Recolección de Muestras}

El material de investigación se recolectó en el departamento de Antioquia, Colombia. Cinco machos bovinos (Bos indicus y Bos taurus) entre tres y cinco años fueron usados para la colecta de semen mediante el método de electroeyaculación (ElectroJac5, Neogen Corporation, USA). Se obtuvieron tres eyaculados por animal para un total de 15 eyaculados. Los animales estuvieron sometidos a condiciones estándar de alimentación, ambiente, manejo reproductivo, y presentaron una condición corporal entre 3 y 4 (escala de 1 a 5). Se estableció un periodo de descanso sexual de una semana antes de cada colecta de semen. Con una cinta escrotal se evaluó la circunferencia escrotal de cada toro al nivel del diámetro mayor de ambos testículos. Se evaluó el volumen de cada eyaculado utilizando una probeta graduada y la concentración espermática mediante un fotómetro Spermacue ${ }^{\circledR}$ (Minitube, Tiefenbach, Alemania). El Comité Institucional para el Cuidado y Uso de Animales de la Universidad CES (CICUA), en su sesión número 38 del 18 de noviembre de 2019 aprobó los protocolos experimentales del presente estudio (Proyecto N. ${ }^{\circ} .200$ ).

\section{Dilución y Criopreservación del Semen}

Cada eyaculado fue dividido en cuatro alícuotas que fueron diluidas en un medio compuesto por Triladyl ${ }^{\circledR}$ (Minitube, Tiefenbach, Alemania) con $8 \% \mathrm{v} / \mathrm{v}$ de lipoproteínas de baja densidad (LDL) (Moussa et al., 2002), y suplementado con caseinato de sodio (CS) mediante cuatro tratamientos: $\mathrm{CS} 3 \% \mathrm{p} / \mathrm{v}(\mathrm{CS} 3), \mathrm{CS} 4 \% \mathrm{p} / \mathrm{v}$ (CS4), CS 5\% $\mathrm{p} / \mathrm{v}$ (CS5) y sin CS (Control). La concentración espermática final para todos los tratamientos fue de $60 \times 10^{6}$ espermatozoides $/ \mathrm{ml}$. 
El semen se empacó en pajillas para 0.5 $\mathrm{ml}$ mediante un equipo automático de empaque y sellado por ultrasonido (MRS1 Dual V2, IMV Technologies, Francia). Las pajillas se mantuvieron a $4{ }^{\circ} \mathrm{C}$ durante $2 \mathrm{~h}$. Posteriormente, se sometieron a vapores de nitrógeno líquido durante 15 minutos y luego fueron sumergidas en un tanque con nitrógeno líquido. Se congelaron tres pajillas por tratamiento a partir de cada eyaculado y toro, para un total de 180 muestras de semen. La descongelación del semen se realizó mediante un baño de agua a $37{ }^{\circ} \mathrm{C}$ durante $60 \mathrm{~s}$.

\section{Evaluación del Semen}

Se evaluó la calidad del semen fresco, diluido y criopreservado para cada uno de los tratamientos establecidos. La movilidad y la cinética espermática se evaluaron mediante el sistema computarizado Sperm Class Analizer (SCA ${ }^{\circledR}$, Microptic SL, España). Se utilizó un microscopio de contraste de fase (Nikon E200, Japón) con una cámara digital (Scout SCA780, Basler, USA). Se analizó la movilidad total (MT), la movilidad progresiva (MP), la velocidad curvilínea (VCL), la velocidad media (VAP), la velocidad lineal (VSL), la amplitud del desplazamiento lateral de la cabeza (ALH) y la frecuencia de batido (BCF) (Varela et al., 2020b). La integridad funcional de la membrana plasmática de los espermatozoides se evaluó la mediante la prueba hipoosmótica (HOS) (Neild et al., 1999).

Adicionalmente, se evaluó la morfología normal $(\mathrm{MN})$ y la vitalidad espermática (VE) del semen fresco y diluido mediante la tinción con eosina-nigrosina (Barth y Oko, 1989), y la integridad estructural de la membrana (IEM) y la integridad acrosómica (IA) del semen descongelado mediante microscopía de fluorescencia.

La IEM se evaluó mediante el kit Live/ Dead (Thermo Fisher Scientific, USA). Se preparó una suspensión de espermatozoides a una concentración de $20 \times 10^{6}$ espermatozoides/ml en solución Hanks Hepes (HH) suplementada con $1 \%$ de albúmina sérica bovina (BSA). Dicha suspensión se incubó por $5 \mathrm{~min}$ a $35^{\circ} \mathrm{C}$ con $6 \mathrm{mM}$ de SYBR-14 y luego otros $5 \mathrm{~min}$ con $0.48 \mathrm{mM}$ de yoduro de propidio. Se evaluaron los patrones de fluorescencia mediante un microscopio E200 con fluorescencia HBO (Nikon, Japón).

La IA de los espermatozoides se evaluó mediante el uso de la sonda fluorescente FITC-PNA (Sigma, USA) (Rathi et al., 2001). Se realizó un extendido de cada muestra espermática, el cual se fijó por 10 minutos con etanol al 95\%. Luego, se dejó secar a temperatura ambiente y se agregaron $25 \mu 1$ de $5 \mu \mathrm{g} / \mathrm{ml}$ de FITC-PNA en solución buffer fosfato (PBS). Cada extendido se guardó en oscuridad por 30 min y se lavó con agua destilada. Se evaluaron 200 espermatozoides usando un microscopio E200 con fluorescencia HBO (Nikon, Japón).

\section{Evaluación de la Capacidad Fertilizante in vitro del Semen Descongelado}

Se colectaron ovarios bovinos en la planta de beneficio de la Central Ganadera de Antioquia (Medellín, Colombia), se depositaron en solución salina $(0.9 \% \mathrm{NaCl})$ a $34{ }^{\circ} \mathrm{Cy}$ se transportaron en termos durante $30 \mathrm{~min}$. Se colocaron en un baño serológico a $30^{\circ} \mathrm{C}$, se aspiraron los folículos de 2 a $8 \mathrm{~mm}$ con una jeringa de $5 \mathrm{ml}$ y una aguja 18G. El líquido folicular se decantó por $15 \mathrm{~min}$ a $30^{\circ} \mathrm{C}$ en un tubo cónico de $50 \mathrm{ml}$ y el pellet se empleó para seleccionar los oocitos grado 1 y 2 según criterios de calidad previamente establecidos (Quispe et al., 2018).

Para la maduración in vitro, los oocitos se lavaron en medio B199, compuesto de TCM 199 y bicarbonato de sodio $(25 \mathrm{mM})$, suplementado con $0.2 \mathrm{mM}$ de piruvato de sodio, $10 \mu \mathrm{g} / \mathrm{ml}$ de sulfato de gentamicina y $10 \%$ de suero fetal bovino, y enriquecido con $0.5 \mu \mathrm{g} / \mathrm{ml}$ de FSH, $5.0 \mu \mathrm{g} / \mathrm{ml}$ de LH y $1 \mu \mathrm{g} / \mathrm{ml}$ de estradiol. Se transfirieron a cajas de Petri, que contenían gotas de $70 \mu 1$ de medio B199 $\mathrm{pH}$ de 7.4. Se colocaron 10 oocitos/gota, se agregaron $3.5 \mathrm{ml}$ de aceite mineral y se incubaron por $24 \mathrm{~h}$ a $38.5^{\circ} \mathrm{C}, 95 \%$ humedad relativa y $5 \%$ de $\mathrm{CO}_{2}$. 
La fertilización in vitro de los oocitos se realizó con espermatozoides bovinos seleccionados por el método del gradiente de Percoll $^{\circledR}$ (Sigma, USA). Se emplearon $250 \mu 1$ de Percoll $90 \%$ y $250 \mu 1$ de Sperm-TALP, que se mezclaron suavemente. El gradiente se elaboró colocando $500 \mu 1$ de Percoll $90 \%$ y sobre este, $500 \mu 1$ de Percoll 45\%. Se incubaron a $38.8^{\circ} \mathrm{C}$ y se depositó $500 \mu \mathrm{l}$ de semen descongelado sobre las dos capas de Percoll. Se centrifugó a 300 g durante $20 \mathrm{~min}$. Se recuperó el precipitado mientras el Percoll fue eliminado. Para cada tratamiento se realizaron entre 3 y 5 repeticiones (según la disponibilidad de oocitos por proceso), donde cada gota con 10 oocitos, se tomó como una repetición.

Se preparó el medio Fert-TALP, suplementado con $10 \mu \mathrm{l} / \mathrm{ml}$ de solución antibiótica, $2 \mathrm{U} / \mathrm{ml}$ de heparina, $2 \mathrm{mM}$ penicilamina, 1 $\mathrm{mM}$ hipotaurina, $250 \mathrm{mM}$ epinefrina, $1 \mathrm{mM}$ piruvato y $6 \mathrm{mg} / \mathrm{ml}$ albúmina sérica bovina (BSA). En cajas de Petri se colocaron gotas de $70 \mu 1$, donde inicialmente se agregó $35 \mu 1$ de medio de fertilización, $3.5 \mathrm{ml}$ de aceite mineral y $35 \mu 1$ de medio. Previo a la coincubación de oocitos con espermatozoides, se evaluó su estadio de maduración por medio de la visualización de la expansión de las células del cúmulus (Restrepo y Vásquez, 2009). Se tomaron $5 \mu 1$ de cada muestra de semen y se diluyó en agua destilada para la evaluación de la concentración. Se ajustó el volumen requerido para obtener una concentración de un millón de espermatozoides/gota. Se coincubaron los oocitos y los espermatozoides por $24 \mathrm{~h}$. Para cada proceso se realizaron grupos control de oocitos sin espermatozoides para estimar partenogénesis.

El cultivo in vitro se realizó en el medio de desarrollo CR-1aa compuesto por 114.6 $\mathrm{mM} \mathrm{NaCl}, 3.08 \mathrm{mM} \mathrm{KCl}, 26.18 \mathrm{mM}$ $\mathrm{NaHCO}_{3}, 2.52 \mathrm{mM}$ lactato de sodio, 0.55 $\mathrm{mM}$ L-lactato, $0.4 \mathrm{mM}$ piruvato de sodio, 1.5 $\mathrm{mg} / \mathrm{ml}$ glutamina, $3 \mathrm{mg} / \mathrm{ml}$ BSAFAF, $1 \%$ solución de antibiótico antimicótico, $10 \mu \mathrm{l} / \mathrm{ml}$ MEM esencial y $20 \mu 1 / \mathrm{ml}$ BME aminoácidos no esenciales. Se eliminaron las células del cúmulus de los oocitos por pipeteo, los presuntos cigotos se lavaron tres veces y se trasladaron a medio de cultivo (día 1), empleando gotas de $100 \mu \mathrm{l}$ cubiertas de $3.5 \mathrm{ml}$ de aceite. Luego se colocaron nuevamente en la incubadora, en las mismas condiciones hasta el día 7. En este periodo se evaluó el clivaje, el cual se ajustó al restar el porcentaje de partenogénesis, y la producción final de blastocistos.

\section{Análisis Estadístico}

Se ajustaron modelos mixtos para cada variable de calidad seminal y para la tasa de producción in vitro de embriones (variables dependientes), en los cuales se incluyó el efecto fijo del tratamiento y el efecto aleatorio del eyaculado anidado dentro del toro. El modelo estadístico fue: $\mathrm{Y}_{i j k l}=\mu+\mathrm{Tto}_{i}+\mathrm{Ri}_{j}+$ Tor $(\mathrm{Ey})_{k}+\mathrm{e}_{i j k l}$, donde $\mathrm{Y}_{i j k l m n}$ : Variable dependiente (parámetro de calidad seminal o producción de embriones), $\mu$ : Media, Tto: Efecto fijo del tratamiento ( $i$ ). ( $i=$ Control, CS3, CS4, CS5), Ri: Efecto fijo de la repetición o pajilla $(j)(j=1 \ldots .5)$, Tor $(\text { Ey })_{k}$ : Efecto aleatorio anidado del eyaculado dentro del toro $(k), \mathrm{e}_{\mathrm{ijkl}}$ : Error aleatorio.

La normalidad de los datos se evaluó mediante la prueba de Shapiro-Wilk. La comparación de las medias se realizó con la prueba de Tukey. Para el análisis de la capacidad fertilizante in vitro se realizó una prueba de Kruskal-Wallis. Se estableció un nivel de significancia de $p<0.05$. Los resultados se expresan como media \pm error estándar de la media. Todas las evaluaciones se realizaron con el programa SAS 9.2. (SAS Inst., USA).

\section{Resultados}

La circunferencia escrotal fue de 39.9 $\pm 0.52 \mathrm{~cm}$. Los resultados para los parámetros de calidad del semen fresco se presentan en el Cuadro 1. El volumen y la concentración de espermatozoides fueron los parámetros de calidad seminal de mayor variabilidad. 
Cuadro 1. Calidad de semen fresco de cinco toros (Bos indicus y Bos taurus) de 3-5 años (Medellín. Colombia)

\begin{tabular}{ccccccc}
\hline Variable & Media & DE & CV & EE & Máximo & Mínimo \\
\hline Volumen $(\mathrm{ml})$ & 7.8 & 4.7 & 60.9 & 0.6 & 18.0 & 3.0 \\
Concentración $(\mathrm{x} \mathrm{10} / \mathrm{ml})$ & 1072.14 & 588.0 & 54.8 & 78.5 & 2360.0 & 408.0 \\
MT $(\%)$ & 91.9 & 12.0 & 13.1 & 1.6 & 99.67 & 41.27 \\
MP $(\%)$ & 75.7 & 16.8 & 22.2 & 2.2 & 94.66 & 21.54 \\
VCL $(\mu \mathrm{m} / \mathrm{s})$ & 83.9 & 18.4 & 21.9 & 2.4 & 121.13 & 37.77 \\
VAP $(\mu \mathrm{m} / \mathrm{s})$ & 49.8 & 11.5 & 23.1 & 1.5 & 71.87 & 23.79 \\
VSL $(\mu \mathrm{m} / \mathrm{s})$ & 34.5 & 9.0 & 26.0 & 1.2 & 55.27 & 14.58 \\
ALH $(\mu \mathrm{m})$ & 3.5 & 0.6 & 19.2 & 0.1 & 5.1 & 1.97 \\
BCF $(\mathrm{Hz})$ & 8.2 & 1.5 & 19.3 & 0.2 & 10.8 & 4.35 \\
HOS $(\%)$ & 78.9 & 10.2 & 13.0 & 1.3 & 96.0 & 47.0 \\
VE $(\%)$ & 85.8 & 5.7 & 6.7 & 0.7 & 94.0 & 66.0 \\
MN $(\%)$ & 84.0 & 6.2 & 7.4 & 0.8 & 95.0 & 62.0 \\
\hline
\end{tabular}

DE: desviación estándar: $\mathrm{CV}$ : coeficiente de variación; EE: error estándar; $\mathrm{MT}$ : movilidad total; MP: movilidad progresiva; VCL: velocidad curvilínea; VAP: velocidad media; VSL: velocidad lineal; ALH: amplitud del desplazamiento lateral de la cabeza; BCF: frecuencia de batido; HOS: integridad funcional de la membrana plasmática; VE: vitalidad espermática; MN: morfología normal

Cuadro 2. Calidad de semen bovino diluido en presencia de caseinato de sodio

\begin{tabular}{ccccccccccc}
\hline & MT & MP & VCL & VAP & VSL & ALH & BCF & HOS & VE & MN \\
\hline Control & $92.5 \pm$ & $74.4 \pm$ & 83.9 & 50.7 & $35.1 \pm$ & $3.5 \pm$ & $7.8 \pm$ & $78.2 \pm$ & $84.6 \pm$ & $86.7 \pm$ \\
& $1.8^{\mathrm{a}}$ & $3.6^{\mathrm{a}}$ & $\pm 5.1^{\mathrm{a}}$ & $\pm 3.4^{\mathrm{a}}$ & $2.7^{\mathrm{a}}$ & $0.1^{\mathrm{a}}$ & $0.4^{\mathrm{a}}$ & $1.8^{\mathrm{a}}$ & $1.9^{\mathrm{a}}$ & $1.3^{\mathrm{a}}$ \\
$\mathrm{CS} 3$ & $96.0 \pm$ & $82.3 \pm$ & 90.0 & 54.3 & $38.3 \pm$ & $3.6 \pm$ & $8.8 \pm$ & $79.5 \pm$ & $86.0 \pm$ & $82.5 \pm$ \\
& $1.2^{\mathrm{a}}$ & $2.5^{\mathrm{a}}$ & $\pm 4.3^{\mathrm{a}}$ & $\pm 2.3^{\mathrm{a}}$ & $1.75^{\mathrm{a}}$ & $0.2^{\mathrm{a}}$ & $0.2^{\mathrm{a}}$ & $2.6^{\mathrm{ab}}$ & $1.4^{\mathrm{a}}$ & $2.0^{\mathrm{a}}$ \\
$\mathrm{CS} 4$ & $85.5 \pm$ & $67.7 \pm$ & 79.6 & 46.2 & $31.4 \pm$ & $3.4 \pm$ & $7.8 \pm$ & $75.7 \pm$ & $85.0 \pm$ & $83.5 \pm$ \\
& $5.3^{\mathrm{a}}$ & $6.5^{\mathrm{a}}$ & $\pm 5.8^{\mathrm{a}} \pm 3.7^{\mathrm{a}}$ & $2.9^{\mathrm{a}}$ & $0.1^{\mathrm{a}}$ & $0.5^{\mathrm{a}}$ & $3.9^{\mathrm{b}}$ & $1.3^{\mathrm{a}}$ & $1.7^{\mathrm{a}}$ \\
$\mathrm{CS} 5$ & $93.5 \pm$ & $78.3 \pm$ & 82.1 & 48.0 & $33.4 \pm$ & $3.5 \pm$ & $8.4 \pm$ & $82.2 \pm$ & $87.7 \pm$ & $83.2 \pm$ \\
& $2.4^{\mathrm{a}}$ & $3.7^{\mathrm{a}}$ & $\pm 4.2^{\mathrm{a}} \pm 2.3^{\mathrm{a}}$ & $1.7^{\mathrm{a}}$ & $0.1^{\mathrm{a}}$ & $0.4^{\mathrm{a}}$ & $2.0^{\mathrm{a}}$ & $1.4^{\mathrm{a}}$ & $1.2^{\mathrm{a}}$ \\
\hline
\end{tabular}

CS3: caseinato de sodio al 3\% p/v; CS4: caseinato de sodio al $4 \% \mathrm{p} / \mathrm{v}$; CS5: caseinato de sodio al $5 \% \mathrm{p} / \mathrm{v}$ MT: movilidad total (\%); MP: movilidad progresiva (\%); VCL: velocidad lineal $(\mu \mathrm{m} / \mathrm{s})$; VAP: velocidad media $(\mu \mathrm{m} / \mathrm{s}) ;$ VSL: velocidad lineal $(\mu \mathrm{m} / \mathrm{s}) ;$ ALH: amplitud del desplazamiento lateral de la cabeza $(\mu \mathrm{m})$; BCF: frecuencia de batido (Hz); HOS: integridad funcional de la membrana plasmática (\%); VE: vitalidad espermática (\%); MN: morfología normal (\%)

$a, b$ Letras diferentes en la misma columna indica diferencia estadística significativa $(p<0.05)$ 
Cuadro 3. Calidad posdescongelación del semen bovino congelado con el empleo de caseinato de sodio como crioprotector

\begin{tabular}{ccccccccccc}
\hline & MT & MP & VCL & VAP & VSL & ALH & BCF & HOS & IEM & IA \\
\hline Control & $62.7 \pm$ & $28.9 \pm$ & $45.3 \pm$ & $27.4 \pm$ & $19.7 \pm$ & $2.0 \pm$ & $4.6 \pm$ & $47.3 \pm$ & $39.6 \pm$ & $98.2 \pm$ \\
& $3.2^{\mathrm{b}}$ & $2.7^{\mathrm{ab}}$ & $2.3^{\mathrm{a}}$ & $1.8^{\mathrm{ab}}$ & $1.5^{\mathrm{ab}}$ & $0.0^{\mathrm{a}}$ & $0.2^{\mathrm{b}}$ & $3.2^{\mathrm{a}}$ & $1.8^{\mathrm{a}}$ & $0.2^{\mathrm{a}}$ \\
CS3 & $68.9 \pm$ & $33.0 \pm$ & $44.7 \pm$ & $29.2 \pm$ & $22.3 \pm$ & $1.9 \pm$ & $5.4 \pm$ & $49.6 \pm$ & $38.9 \pm$ & $98.3 \pm$ \\
& $2.8^{\mathrm{a}}$ & $2.2^{\mathrm{a}}$ & $1.4^{\mathrm{a}}$ & $1.2^{\mathrm{a}}$ & $1.0^{\mathrm{a}}$ & $0.0^{\mathrm{ab}}$ & $0.2^{\mathrm{a}}$ & $3.3^{\mathrm{a}}$ & $1.6^{\mathrm{a}}$ & $0.2^{\mathrm{a}}$ \\
$\mathrm{CS} 4$ & $48.2^{2} \pm$ & $20.0 \pm$ & $38.5 \pm$ & $23.3 \pm$ & $17.4 \pm$ & $1.8 \pm$ & $4.5 \pm$ & $37.1 \pm$ & $30.9 \pm$ & $97.3 \pm$ \\
& $3.6^{\mathrm{c}}$ & $2.3^{\mathrm{c}}$ & $1.8^{\mathrm{b}}$ & $1.5^{\mathrm{b}}$ & $1.3^{\mathrm{b}}$ & $0.0^{\mathrm{b}}$ & $0.2^{\mathrm{b}}$ & $2.5^{\mathrm{b}}$ & $1.9^{\mathrm{b}}$ & $0.3^{\mathrm{a}}$ \\
CS5 & $58.5 \pm$ & $25.5 \pm$ & $38.7 \pm$ & $23.8 \pm$ & $17.7 \pm$ & $1.8 \pm$ & $4.8 \pm$ & $45.0^{2} \pm$ & $38.9 \pm$ & $98.0 \pm$ \\
& $3.2^{\mathrm{b}}$ & $2.0^{\mathrm{b}}$ & $1.2^{\mathrm{b}}$ & $0.9^{\mathrm{b}}$ & $0.9^{\mathrm{b}}$ & $0.0^{\mathrm{b}}$ & $0.2^{\mathrm{ab}}$ & $3.1^{\mathrm{a}}$ & $2.2^{\mathrm{a}}$ & $0.3^{\mathrm{a}}$ \\
\hline
\end{tabular}

CS3: caseinato de sodio al 3\% p/v; CS4: caseinato de sodio al 4\% p/v; CS5: caseinato de sodio al 5\% p/v MT: movilidad total (\%); MP: movilidad progresiva (\%); VCL: Velocidad curvilínea ( $\mu \mathrm{m} / \mathrm{s})$; VAP: Velocidad medio $(\mu \mathrm{m} / \mathrm{s})$; VSL: velocidad lineal $(\mu \mathrm{m} / \mathrm{s}) ; A L H$ : amplitud del desplazamiento lateral de la cabeza $(\mu \mathrm{m})$; BCF: Frecuencia de batido ( $\mathrm{Hz})$; HOS: Integridad funcional de membrana (\%); IEM: integridad estructural de membrana (\%); IA: Integridad acrosómica (\%)

Los resultados son presentados como la media \pm error estándar de la media (SEM)

$a, b$ Letras diferentes en la misma columna indica diferencia estadística significativa $(p<0.05)$

El efecto aleatorio del eyaculado anidado dentro del toro fue significativo para todos los modelos ajustados $(\mathrm{p}<0.05)$, tanto para el semen diluido como para el semen criopreservado, lo que sugiere que la variabilidad existente en la calidad del semen se puede atribuir en gran medida al reproductor e incluso a los eyaculados de un mismo animal. La adición de caseinatos en la etapa de dilución del semen bovino no produjo mayores efectos en la calidad seminal excepto por la reducción de la HOS para el tratamiento CS4 en comparación con C5 ( $\mathrm{p}<0.05)$ (Cuadro 2).

En el semen congelado y descongelado se observó un incremento en la MT y la BCF por efecto del tratamiento CS3, mientras que CS4 y CS5 produjeron una reducción en diferentes parámetros relacionados con la movilidad, la cinética y la integridad de membrana $(\mathrm{p}<0.05)$ (Cuadro 3). Todos los modelos ajustados para la evaluación del semen descongelado presentaron coeficientes de de- terminación $\left(\mathrm{R}^{2}\right)$ mayores a 0.70 , por lo que se infiere que los efectos considerados en el estudio explicaron en una alta proporción la variabilidad de cada parámetro. En cuanto a la capacidad fertilizante in vitro del semen congelado y descongelado, no se encontraron diferencias entre los tratamientos $(\mathrm{p}>0.05)$ (Figura 1).

\section{Discusión}

El uso de leche entera y leche descremada en los diluyentes de congelación para proteger los espermatozoides durante el almacenamiento ha sido ampliamente descrito (Bergeron et al., 2007; Lusignan et al. 2011). La leche entera contiene lipoproteínas que podrían tener un efecto crioprotector, en tanto que la leche descremada no las tiene; sin embargo, es igualmente eficaz para proteger los espermatozoides (Plante et al., 2015). Con base a esto, se ha postulado que las proteínas 


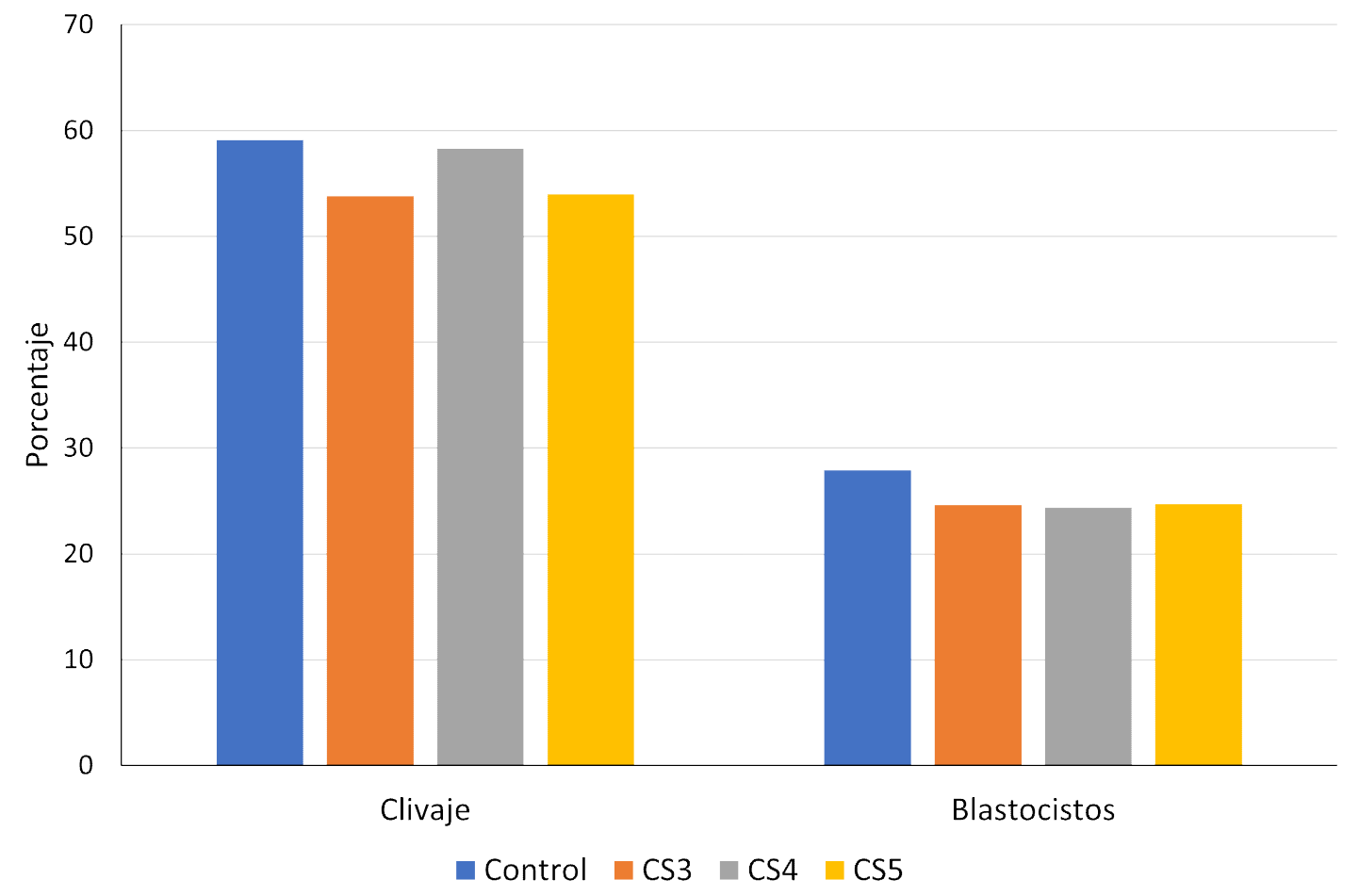

Figura 1. Capacidad fertilizante in vitro de semen bovino congelado con caseinato de sodio. CS3: caseinato de sodio al 3\% $\mathrm{p} / \mathrm{v}$. CS4: caseinato de sodio al $4 \% \mathrm{p} / \mathrm{v}$. CS5: caseinato de sodio al $5 \% \mathrm{p} / \mathrm{v}(p>0.05)$

de la leche, las caseínas, confieren el efecto crioprotector (Lusignan et al., 2011). Dichas proteínas forman fácilmente películas en soluciones acuosas debido a su estructura desordenada y a su capacidad de formar gran número de puentes de hidrógeno e interacciones y puentes hidrofóbicos (Bergeron et al., 2007; Lusignan et al., 2011).

En el presente estudio la dilución del semen bajo los tratamientos con CS tuvo un efecto equivalente al tratamiento control, con relación con la movilidad, cinética, integridad de membrana, vitalidad y morfología; sin embargo, CS4 redujo HOS respecto al tratamiento CS5, aunque sin otros impactos significativos sobre la cinética espermática (Cuadro 2). En el semen congelado y descongelado, tanto CS4 como CS5 produjeron efectos deletéreos en múltiples parámetros, siendo llamativo que CS4 afectó tanto la movilidad como la integridad y la funcionalidad de la membrana plasmática (Cuadro 3). Esto podría estar relacionado con el efecto osmótico producido por la adición de mayores concentraciones de CS, que pudiese generar una condición de estrés osmótico y que, además, pudo verse exacerbada por el proceso de congelación, con un consecuente daño mecánico y oxidativo sobre la membrana plasmática y la alteración del metabolismo espermático (Meyers, 2005).

No obstante, el tratamiento CS3 incrementó la MT y la BCF del semen descongelado en comparación con el tratamiento control, lo cual, además de no asociarse con posibles alteraciones de origen osmótico u oxidativo, podría atribuirse al efecto benéfico de las caseínas aisladas de la leche mediante su interacción con las proteínas del plasma seminal bovino (BSP) (Bergeron et al., 
2007; Plante et al., 2015). Las BSP son conocidas como perjudiciales para el propósito de la congelación seminal por su capacidad para extraer el colesterol y los fosfolípidos de la membrana plasmática (Bergeron y Manjunath 2006; Manjunath et al., 2007). La interacción de las micelas de caseína con las proteínas BSP impide dicha extracción, de manera similar a la acción protectora de las lipoproteínas de baja densidad (LDL) de la yema de huevo, manteniendo la viabilidad y la movilidad de los espermatozoides durante su almacenamiento (Bergeron et al., 2007; Lusignan et al., 2011). Por otro lado, las LDL purificadas han permitido por sí solas alcanzar altos niveles de protección espermática durante la congelación (Moussa et al., 2002); sin embargo, estudios previos de este grupo de investigación muestran que, aunque estas tienen un efecto equivalente a la yema de huevo entera y la yema de huevo centrifugada en la conservación de la actividad mitocondrial, la integridad y la estabilidad de la membrana plasmática, no alcanzan el nivel de conservación de la MP de la yema de huevo centrifugada (Varela-Giraldo et al., 2020; Varela et al., 2020a). Por lo tanto, el mejoramiento observado de la MP por la adición de CS en un diluyente suplementado con LDL, otorga un nivel de protección espermática adicional, en un medio completamente definido con estos dos componentes purificados de origen animal.

Estudios previos han demostrado que el uso de diluyentes sin caseínas y yema de huevo bajo diferentes periodos y temperaturas de almacenamiento producen entre el $10 \mathrm{y}$ $15 \%$ de pérdida de lípidos de la membrana plasmática (Bergeron et al., 2004, 2007). Esto podría deberse a la concentración de plasma seminal en el semen diluido, ya que la eliminación de lípidos de la membrana por efecto del plasma seminal o sus proteínas BSP depende de dicha concentración (Manjunath y Thérien, 2002; Bergeron et al., 2007). En relación con los resultados del presente estudio, la proporción de plasma seminal y la concentración de proteínas BSP podrían ser factores asociados a la variabilidad encontrada en la calidad espermática atribuible a los efectos del eyaculado y el reproductor.

En cuanto a la capacidad fertilizante in vitro, no se evidenciaron diferencias entre los tratamientos (Figura 1). Sin embargo, se pudo deducir que el semen bovino congelado en un diluyente a base de tris-glicerol-ácido cítrico y LDL, con o sin CS, conserva su capacidad posdescongelación para fertilizar oocitos y desarrollar embriones en condiciones in vitro. Se conoce que las caseínas modulan de forma positiva la inducción de la capacitación espermática, proceso esencial para la fertilización (Manjunath y Thérien, 2002). Otro estudio donde se evaluó el uso de diluyentes alternativos con ciclodextrinas y colesterol para la congelación de semen bovino reportó igualmente la conservación de la capacidad fertilizante in vitro de los espermatozoides (Anzar et al., 2019).

\section{Conclusiones}

- La suplementación del diluyente de congelación con caseinato de sodio puede mejorar la calidad posdescongelación del semen bovino, dado que en una concentración del 3\% $(\mathrm{p} / \mathrm{v})$ tiene un impacto positivo sobre la movilidad y la cinética espermática.

- El uso de caseinato de sodio en concentraciones superiores al 3\% (p/v) en el diluyente de congelación tiene efectos deletéreos posdescongelación sobre la movilidad, la cinética y la integridad estructural y funcional de la membrana plasmática de los espermatozoides bovinos.

- El uso de un diluyente de congelación de semen bovino basado en componentes de origen animal definidos como caseinato de sodio y LDL, podría constituirse en una alternativa a los diluyentes con componentes de origen animal no purificados y otros suplementos de origen vegetal. 


\section{Agradecimientos}

A la Universidad CES, Medellín, Colombia, por la financiación para la realización de esta investigación y al personal del Laboratorio de Biotecnología Animal del Politécnico Colombiano Jaime Isaza Cadavid, Medellín, Colombia, por su apoyo en la fase experimental de este estudio.

\section{Literatura Citada}

1. Aires VA, Hinsch KD, MuellerSchloesser F, Bogner K, MuellerSchloesser S, Hinsch E. 2003. In vitro and in vivo comparison of egg yolkbased and soybean lecithin-based extenders for cryopreservation of bovine semen. Theriogenology 60: 269-279. doi:10.1016/S0093-691X(02)01369-9.

2. Anzar M, Rajapaksha K, Boswall L. 2019. Egg yolk-free cryopreservation of bull semen. PLoS One 14: e0223977. doi: 10.1371/journal.pone.0223977

3. Barth AD, Oko RJ. 1989. Abnormal morphology of bovine spermatozoa. USA: Iowa University Press. 285 p.

4. Batellier F, Magistrini M, Fauquant J, Palmer E. 1997. Effect of milk fractions on survival of equine spermatozoa. Theriogenology 48: 391-410. doi: 10.1016/S0093-691X(97)00250-1

5. Bergeron A, Brindle Y, Blondin P, Manjunath P. 2007. Milk caseins decrease the binding of the major bovine seminal plasma proteins to sperm and prevent lipid loss from the sperm membrane during sperm storage. Biol Reprod 77: 120-126. doi: 10.1095/ biolreprod.106.058248

6. Bergeron A, Crête MH, Brindle Y, Manjunath P. 2004. Low-density lipoprotein fraction from hen's egg yolk decreases the binding of the major proteins of bovine seminal plasma to sperm and prevents lipid efflux from the sperm Membrane. Biol Reprod 70: 708717. doi:1 0.1095/biolreprod.103.022996
7. Bergeron A, Manjunath P. 2006. New insights towards understanding the mechanisms of sperm protection by egg yolk and milk. Mol Reprod Dev 73: 13381344. doi: $10.1002 / \mathrm{mrd} .20565$

8. Quispe C, Ancco E, Solano J, Unchupaico I, Mellisho E. 2018. Embryonic development capacity of bovine oocytes aspired by ovum pick-up and from slaughterhouse ovaries. Rev Inv Vet Perú 29: 1114-1121. doi:10.15381/ rivep.v29i4.14418

9. Kumar P, Saini M, Kumar D, Balhara AK, Yadav SP, Singh P, Yadav PS. 2015. Liposome-based semen extender is suitable alternative to egg yolk-based extender for cryopreservation of buffalo (Bubalus bubalis) semen. Anim Reprod Sci 159: 38-45. doi: 10.1016/j.anireprosci.2015.05.010

10. Layek SS, Mohanty TK, Kumaresan A, Parks JE. 2016. Cryopreservation of bull semen: evolution from egg yolk based to soybean based extenders. Anim Reprod Sci 172: 1-9. doi: 10.1016/j.anireprosci.2016.04.013

11. Lusignan MF, Bergeron A, Lafleur M, Manjunath P. 2011. The major proteins of bovine seminal plasma interact with caseins and whey proteins of milk extender. Biol Reprod 85: 457-464. doi:10.1095/biolreprod.110.089961

12. Manjunath P, Bergeron A, Lefebvre J, Fan J. 2007. Seminal plasma proteins: functions and interaction with protective agents during semen preservation. Soc Reprod Fertil Suppl 65: 217-228.

13. Manjunath P, Thérien I. 2002. Role of seminal plasma phospholipid-binding proteins in sperm membrane lipid modification that occurs during capacitation. J Reprod Immunol 53: 109-119. doi: 10.1016/S0165-0378(01)00098-5

14. Meyers SA. 2005. Spermatozoal response to osmotic stress. Anim Reprod Sci 89: 57-64. doi: 10.1016/j.anireprosci.2005.06.026 
15. Moussa M, Martinet V, Trimeche A, Tainturier D, Anton M. 2002. Low density lipoproteins extracted from hen egg yolk by an easy method: cryoprotective effect on frozen-thawed bull semen. Theriogenology 57: 1695-1706. doi: 10.1016/S0093-691X(02)00682-9

16. Murphy EM, Murphy C, O'Meara C, Dunne G, Eivers B, Lonergan P, Fair S. 2017. A comparison of semen diluents on the in vitro and in vivo fertility of liquid bull semen. J Dairy Sci 100: 15411554. doi: $10.3168 /$ jds.2016-11646

17. Neild D, Chaves G, Flores M, Mora N, Beconi M, Agüero A. 1999. Hypoosmotic test in equine spermatozoa. Theriogenology 51: 721-727. doi: 10.1016/S0093-691X(99)00021-7

18. Plante G, Lusignan MF, Lafleur M, Manjunath P. 2015. Interaction of milk proteins and binder of sperm (BSP) proteins from boar, stallion and ram semen. Reprod Biol Endocrin 13: 92. doi: 10.1186/s12958-015-0093-1

19. Pugliesi G, de Carvalho GR, Rates $D M$, Ker PG, da Matta MP, de Oliveira RR, da Silva Filho JM. 2012. Viability and fertility of cooled equine semen diluted with skimmed milk or glycine egg yolk-based extenders. Rev Bras Zootec 41: 2411-2417. doi: 10.1590/ S1516-35982012001200005

20. Rathi R, Colenbrander B, Bevers MM, Gadella BM. 2001. Evaluation of in vitro capacitation of stallion spermatozoa. Biol Reprod 65: 462-470. doi: 10.1095/ biolreprod65.2.462

21. Restrepo G, Vásquez N. 2009. Efecto de la maduración in vitro de oocitos bovinos con suero fetal bovino sobre su actividad mitocondrial post-desvitrificación. Rev MVZ Córdoba 14: 15541562. doi: $10.21897 / \mathrm{rmvz} .364$

22. Röpke T, Oldenhof H, Leiding C, Sieme H, Bollwein H, Wolkers WF. 2011. Liposomes for cryopreservation of bovine sperm. Theriogenology 76:
1465-1472. doi:10.1016/j.theriogenology.2011.06.015

23. Singh AK, Singh VK, Narwade BM, Mohanty TK, Atreja SK. 2012. Comparative quality assessment of buffalo (Bubalus bubalis) semen chilled $\left(5^{\circ} \mathrm{C}\right)$ in egg yolk- and soya milk-based extenders. Reprod Domest Anim 47: 596-600. doi: 10.1111/j.1439-0531.2011.01928.x

24. Singh VK, Singh AK, Kumar R, Atreja SK. 2013. Development of soya milk extender for semen cryopreservation of Karan Fries (crossbreed cattle). CryoLetters 34: 52-61.

25. Thibier M, Guerin B. 2000. Hygienic aspects of storage and use of semen for artificial insemination. Anim Reprod Sci 62: 233-251. doi: 10.1016/S03784320(00)00161-5

26. Varela-Giraldo E, Úsuga-Suárez A, Duque-Cortés JE, Gómez-Oquendo J, Restrepo-Betancur G. 2020. Effect of low-density lipoproteins and trehalose on cryopreserved bovine semen quality. Rev Colomb Cienc Pecu 34: 200-211. doi: 10.17533/udea.rccp.v34n3a03

27. Varela E, Rojas M, Restrepo G. 2020a. Membrane stability and mitochondrial activity of bovine sperm frozen with lowdensity lipoproteins and trehalose. Reprod Domest Anim 55: 146-153. doi: 10.1111/rda.13599

28. Varela E, Rojas M, Restrepo G $2020 b$. Association between conventional and computerized sperm quality parameters with flow cytometric evaluation of frozen bovine semen. Rev Inv Vet Perú 31: e19023. doi: 10.15381/Rivep.v31i4.19023

29. Van Wagtendonk-de Leeuw AM, Haring RM, Kaal-Lansbergen LMTE, Den Daas JHG 2000. Fertility results using bovine semen cryopreserved with extenders based on egg yolk and soy bean extract. Theriogenology 54: 57-67. doi: 10.1016/S0093-691X(00)00324-1 